The Public Journal of Semiotics II(2), July 2008, pp. 2-29

\title{
Signs for Language Origins?
}

\author{
Adam Kendon \\ Philadelphia, Pennsylvania \\ adamk@dca.net
}

\begin{abstract}
Taking the recent publication of The Gestural Origin of Language by David Armstrong and Sherman Wilcox as a starting point, this essay discusses a number of issues and difficulties raised by the idea that language first emerged as a gesture-language, only later to become spoken. It is argued that while modern sign languages may throw light on processes that are fundamental to language formation, they cannot be considered representations of an earlier form of language, as some writers seem to suppose, nor does their existence offer any support for a 'gesture first' theory. Rather, language must have been, from its first appearance, a multimodal phenomenon. It is pointed out that modern speakers, qua speakers speaking spontaneously, always employ several modalities together in a complex orchestration. However, the model of language generally followed in linguistics, whether the language studied is spoken or signed, does not usually take this into consideration. An abstracted idea of language is usually employed, developed largely because the systematic study of language usually considers language only in its written form, and not as it is manifested when spoken, when it is an activity that involves the whole body, and not just the so-called 'speech apparatus'.
\end{abstract}

\section{Introduction}

In The Gestural Origin of Language (2007) David Armstrong and Sherman Wilcox argue that by examining the processes by which "visual varieties of language, especially signed languages of the deaf but also writing" come into being "we can learn much about the way language in general probably emerges" (p. 7 ). In this, as the authors acknowledge, they follow a position previously put forward by E. B. Tylor (among others) who, in his Researches into the Early History of Mankind (1865) wrote that through the study of gesture language and picture writing it would be possible to "realise to ourselves in some measure a condition of the human mind which underlies anything which has yet been traced in even the lowest dialect of Language if taken as a whole (p. 15)." That is to say, the study of gesture and picture writing can reveal to us something of the fundamental process by which language is created, which cannot be achieved if we consider only spoken language. This is because, if we examine the history of spoken languages, we can only see how contemporary words derive from other words. If we examine the history of the development of writing and also changes with time of signs in signed languages, on the other hand, we can see how contemporary forms appear to be derivable from earlier forms that do not have the highly coded forms characteristic of the units of language. Thus, in the history of the Sumerian script, for example (which Armstrong and Wilcox refer to in the penultimate chapter of their book), it is possible to observe a progression from earlier pictorial forms to later forms that are highly conventionalised and seemingly arbitrary in form. 
Likewise, in sign languages, as has been described by a number of authors (Tervoort 1961, Frishberg 1975, Bellugi and Klima 1976, among others), when signers introduce a new item into their lexicon they often do this by first referring to the new item by means of a depictive gesture or pantomime. Subsequently, such a form may become simplified and stabilised in form, it takes on characteristics which are shared by other items already in the sign lexicon, establishing itself as a highly 'reduced', conventionalised, even seemingly arbitrary form, which can become shared as a vocabulary item in the community of signers within which it arose. By looking at the emergence of signed languages, thus, we seem to be able to witness the process by which something that at first is not 'linguistic' becomes so.

To be able to do this, of course, requires that we have an idea of what is 'not linguistic' and what is 'linguistic', so that we can understand the trajectory of transformation being examined. The present authors leave this to the reader. They take it for granted that it will be understood what it is that is supposed to have emerged for they nowhere offer an explicit definition of 'language'. From the way the authors discuss things, however, we may gather that they tend to use the word 'language' in two main senses: a functional sense and a formal sense. From the point of view of a functional sense, any sort of action that makes possible discursive reference to concepts or ideas would be counted as 'language'. For example if, by my idiosyncratic pantomiming and vocal growling, I am able to make you understand that there was a panther near the camp last night, this would be an instance of 'language' considered from a functional point of view. How 'language', in this sense, came into being is one kind of language origins question. From the point of view of a formal sense of the word 'language', on the other hand, this discourse about last night's panther visit would not be regarded as 'language' unless the units of action I had used to convey my meaning were highly conventionalised, were arbitrary in respect to how their forms relate to their meanings, and can be analysed into interchangeable components which function only to maintain contrasts between the forms being used. Further, it would be important to show that the way in which these meaning-bearing units are organised in relation to one another can be accounted for in terms of certain more general syntactic rules. How 'language', in this sense, came into being, is another kind of language origins question. Since the authors do not keep separate these different senses of the word 'language' it is not always clear which language origin question they are discussing. So when they refer to the evolutionary development of our abilities to conceptualise objects and interactive processes between objects as leading to the emergence of language (see pp. 90-91), we don't know whether they want us to think that what is emerging here is 'language' in the functional sense distinguished above, or 'language' in the formal sense.

The present authors previously collaborated with the late William Stokoe and, together with him, they had laid out their main argument in a book published in 1995 (Armstrong, Stokoe and Wilcox 1995), Stokoe himself published a book on his own in 2001 which argued along similar lines (Stokoe 2001), while Armstrong published his own separate discussion in 1999 (Armstrong 1999). As in these other publications, so here, the central claim is that it must have been in the medium of visible action that linguistic expression first made its appearance. 
A possible corollary to this claim is that 'sign language' was, in consequence, the first form of language. Some authors have, in fact, made this claim. For example, Corballis (2002: 125) writes that his "guess is that the precursors of H. sapiens had in fact evolved a form of signed language similar in principle, if not in detail, to the signed languages that are used today by the deaf." A similar view was expressed by Hewes (1973). The present authors are perhaps not quite so strongly committed to this idea. On p. 17 they say they start from the premise "that signed languages are the original and prototypical languages", but elsewhere in the book they do not exclude the idea that evolutionarily early forms of language also had a vocal component. Thus, on p. 68 they write "there never was a time when visible gestures were unaccompanied by vocalizations." As will be discussed further below, the issue of when and how spoken language evolved and what its relationship may be to signed language, whether evolutionarily early or modern, remain points of considerable difficulty.

\section{Origins of the 'gesture first' theory}

The idea that human language (at least in the functional sense of this term) can be accomplished by means of visible bodily actions, or 'gestures', as well as by means of vocalisations, is by no means new, of course. Armstrong and Wilcox quote a passage from Plato's Cratylus which makes it clear that the idea that one could accomplish what may be expressed in a spoken language with gestures instead of speech was well known in Classical Antiquity (gesturing deaf-mutes were known in Ancient Greece). It was often supposed, however, that not only could gestural expression serve the same functions as spoken language. It was also seen as more natural, perhaps because it was seen as something that all humans had in common. In this it contrasted with spoken languages, which because they differ radically from one another, seem more like artificial inventions. As Quintillian remarks, writing in the First Century $\mathrm{CE}$, “...though the peoples and nations of the earth speak a multitude of tongues, they share in common the universal language of the hands" (Quintilian 1922: 291). Spoken languages differ widely and promote divisions among humans. By contrast, the language of gesture seems to unite them.

This idea of gesture as a universal and unifying form of expression finds formulation not long after the beginning of the modern era. The first books ever to have been published that were devoted wholly to the topic of gesture were Bonifaccio's Arte de' Cenni (Art of Signs) of 1616 and John Bulwer's treatises Chirologia ... and Chironomia... that were published together in a single volume in 1644 (Bulwer 1974). Both authors proposed that gesture was more natural as a form of expression than spoken language. Bonifaccio wondered if the state of human society would be improved if we all returned to this form of expression, originally given to us by God, because it could be understood by everyone, and Bulwer, likewise, wondered if gesture was a form of language that "had the happiness to escape the curse at the confusion of Babel" (Bulwer 1974:19)

In the eighteenth century the possible natural (as opposed to Divine) origins of language began to be widely discussed. Given the idea that gestural expression was both natural to humans and universal in its form, it is not surprising 
that the idea came to be widely entertained that language began with visible actions, not with vocal utterances. An early explicit expression of this idea, as is mentioned by Armstrong and Wilcox, is to be found in a treatise by William Warburton, Bishop of Gloucester, who had published, between 1738 and 1741, a large book on Egyptian hieroglyphics. He argued that these hieroglyphics were not invented by priests as an obscure form of expression as a means to preserve their power. Rather, they had evolved as abbreviations of an earlier form of picture writing. Writing, according to Warburton, began as a form of pictorial representation, and then, as the ideas to be expressed became more numerous, these representations underwent a process of progressive reduction, until they no longer had any representational features. Only then would they be completely obscure to those who had not studied them. Warburton believed that this process reflected a general process in terms of which the origins of all languages could be explained. In the beginning, he said, humans expressed themselves by pantomimic gestures, by significant actions and expressive sounds. Evidence for this could be found in the accounts of the deeds of the Prophets of the Old Testament, who often were described as engaging in dramatic actions to convey what they had to say. In the course of time, however, these expressions became shortened and transformed into figurative speech and then into the highly abstract expressions derived through reason that characterises modern languages (Rosenfeld 2001: 38-39). These ideas were taken up by Etienne Bonnot de Condillac who, in his treatise on the origins of human knowledge published in 1746, quoted extensively from Warburton's work. He proposed a scenario in which the first interchanges of a linguistic kind would have been carried out through actions rather than through vocal utterances, that expressions would have been combinations of pantomime and vocal expression and only later did the voice become more and more refined until it was capable of the sophistication and complexity of expression of modern times.

At the same time as Condillac was developing these ideas in Paris, and unknown to him, Giambattista Vico, in Naples was writing his Scienza Nuova (the third, final edition of which was published in 1744, the year of his death). In this book he, too, proposed that language began with visible actions. Vico, however, gave emphasis to the idea that language begins as a "mental language" which developed from "poetic logic" in which humans perceived things and events metaphorically. He writes "the first poets attributed to physical bodies the being of animate substances" [404] and he draws attention to the fact that "in all languages most expressions for inanimate objects employ metaphors derived from the human body and its parts, or from human senses and emotions" [405]. Thus a potato is said to have 'eyes', a fork is said to have 'teeth', a peach is said to be composed of 'flesh' outside with a 'bone' inside (and, indeed, it has a 'skin' too). For Vico, this kind of metaphorical perception is fundamental to the establishment of the conceptual categories which a language expresses, which at first is done by way of "signs, 
actions or physical objects which had a natural relation to the ideas expressed [401]." ${ }^{1}$ Language (functionally understood) could have had its origin in this.

This capacity for metaphorical perception, of being able to see abstract resemblances between otherwise unrelated events or objects, a capacity also stressed by Heinz Werner in his account of symbol formation (Werner and Kaplan 1963), is fundamental to the scenario that Armstrong and Wilcox propose by which language first became established. For they suppose that what came about was a capacity to see that movements of the hands could directly represent objects and events in the environment. This idea was put forward in a particularly clear manner by William Stokoe (1991) in a paper much quoted by the present authors. In a critique of what were, in his opinion, misguided attempts to analyse signs in a sign language in terms of models borrowed from phonological analyses of spoken languages, Stokoe pointed out that a sign is also an action. For example, moving the right hand partially open over to the left hand, posed so that the index finger is held vertical and, in the moment of the approach of the right hand its fingers close round the left hand's index finger, to sign CATCH or GRASP, is an action in which the right hand grasps the upraised finger of the left hand. And as an action it already has meaning: the acting hand acts on or in relation to another body part. Further, Stokoe argued, the entire configuration of action contains within itself the structure of an event which could be seen as having a sentential representation: the moving hand is at once an agent and an action. In the sign for CATCH or GRASP the finger the right hand grasps is the 'object' of this 'action'. Within the unit of action which is the 'sign', thus, there is already a structure of agents and objects and actions joining them in some relation. The crucial step, it seems, was the development of an ability to see "hand-shapes representing subjects, their movements representing predication, and the whole gesture a complete thought..." (Stokoe 2001: 82). Or, as Armstrong and Wilcox write: "Visual articulators such as hands and faces come with inherent conceptual significance" (p. 109) adding (on another page): "Visible gestures are at once actions in the world, actions with instrumental function (grasping prey) and, at least potentially, communicative actions, acts that convey information, intent and relationship ("I grasped the prey"). It is not merely that visible gestures can be iconic for objects and events in the world - visible gestures are objects and events in the world." (p. 64).2

\footnotetext{
${ }^{1}$ See the section 'Poetic Logic' in Giambattista Vico New Science (1999, trans. David Marsh. See also Bergin and Fisch 1984). The numbers in square brackets refer to paragraph numbers in Vico's text. For one attempt to systematise Vico's views on language origins and to align these with modern studies see Danesi (1993).

${ }^{2}$ In this they appear to have been anticipated by van Ginneken (1939), at least as his views are described by Todorov (1982). Todorov (p. 234) states that according to van Ginneken the gesture [as a linguistic action] is primordial because "it is part of the action it designates ... the [gesture] sign signifies itself." He quotes van Ginneken (1939:127): “The gesture in this case is nothing but the work begun in the outdoor air, and that the manual concept brings to life again inside. Thus it is natural language. For here there is no convention. The sign is the natural sign, for it is the signified itself." See also the fascinating article by Frank Cushing (1892) who claims that the actions of the hands are intimately involved in the emergence of language, not just of words but of syntax also: “...the very earliest uttered speech was already framed complexly by the two hands - one acted upon, the other acting upon it." He seeks to demonstrate this with an analysis of Zuni verbs.
} 
On this view, then, the first development to take place in the direction of being able to use actions as a means to communicate something about objects and events in the world (rather than as a way of indicating a likelihood of acting in a certain way) - development of 'language' in the functional sense distinguished above - required the ability to see visible actions, motions of the hands, let us say, as being like the motions and interactions of objects in the environment. Engaging in forms of action that are seen as like forms of action that can be observed in the environment provides the very first step in the emergence of language. An understanding of how this kind of perception began phylogenetically is crucial for an understanding of how language (in the functional sense) started. As far as I know, not much is known about this (but see Hurford 2007 for a suggestive review).

However, an ability to see visible actions in terms of their likeness to motions and interactions of objects in the environment would not, in itself, be sufficient to start a language. As Burling (2005) has pointed out, just as important is an ability to represent such perceptions for others. He writes (p. 20) "If no one else was around with the skills to understand, what could the first speaker hope to accomplish with her first words? The puzzle dissolves as soon as we recognize that communication does not begin when someone makes a meaningful vocalization or gesture, but when someone interprets another's behavior as meaningful." Bickerton (1981:264) raises the question of how the actions of another come to be recognised as meaningful: "When A, the first hominid ever to use a sound sequence or a gesture referentially made such a sequence or gesture to B, another hominid, how did B know that A was communicating referentially and not merely coughing, clearing his throat, scratching himself , or brushing a fly away?" (see also Kendon 1991).

In reference to this it has lately become common to refer to the discoveries of mirror neurons which suggest that when an action is observed, neurons in the observer become active that would be used were the observer to carry out that action. This seems to provide a physiological basis for empathy, and has provoked much discussion (Rizzolatti and Arbib 1998, Stamenov and Gallese 2002, Bråten 2007) although, as Damasio and Kaspar (2008) have pointed out, rather little is actually understood about how they actually play a role in the processes of action recognition in others.

\section{Conceptions of language}

Starting a discussion about the origin of language in this manner means that the conception one has of 'language' and of the processes by which it came into being is one that sees it as something that has emerged through processes that, in principle, are not special to it but are involved in many other kinds of cognitive and expressive functions. This is very different from the conception of language that has been dominant in many current discussions of this topic.

Armstrong and Wilcox identify as "Cartesian" the view that what is distinctive about language is its syntactic component, understood as a set of formal algorithms that generate strings of elements in sequences that conform to grammatical rules. It is of central importance to these rules that the sequences of grammat- 
ical strings can have the property of recursion (embedding phrases within phrases) for it is this that makes possible the infinity of expressions that has been identified as the distinguishing character of language, from this point of view. This socalled "Cartesian" view nowadays, of course, is largely identified with the work of Noam Chomsky, which may be said to be its epitome. As is very well known, Chomsky has long maintained that this feature is found nowhere else in the animal kingdom, that it is unique to humans. In consequence he has held out little hope that any light could be thrown on the origin of language through the comparative study of the communication systems of other animals. Hence, for him, the possibility of an explanation of language origins in terms of evolutionary theory seems quite remote. He suggested that humans came to possess this defining feature of language as a result of the intervention of a mutation that brought about a reorganisation of the brain, an idea that has found favour with only a few scholars, since it is not in good accord with modern concepts of the process of biological evolution. Lately, Chomsky has modified his position somewhat. In a recent paper, together with two colleagues who are experts in animal communication, Chomsky has suggested that comparative studies of the cognitive capacities of other animals might throw light on the origin of this syntactic capacity (Hauser, Chomsky and Fitch 2002). However, it is suggested that this capacity might not have developed in relation to language at all, but might be the consequence of some other cognitive capacities (capacities connected to orientation within and exploration of the environment, for example) which came to be "exapted" for language at a certain stage in human evolution. How they came to be so, if indeed this happened, is left unaccounted for.

Armstrong and Wilcox's approach is quite different. ${ }^{3}$ In their view, language is seen as growing "out of the human body interacting with its physical and social environments - metaphorical structures are the pathways from gesture to meaning." (101). Language is an emergent consequence of certain perceptual, cognitive and social interactive processes, that is to say, and it does not require the postulation of specialised modules in the mind (or brain) that are autonomous, separate from cognitive processes that operate in other realms, wholly novel and unique to the human species, or derived from something that has nothing to do with communication. As noted above, following Stokoe, they argue that even the elaborate syntaxes of modern spoken languages can be seen to have their roots in a process by which a syntactic structure can be unpacked from unitary representations of events. In arguing for this view, they make use of the framework of cognitive linguistics, especially as this has been developed by Ronald Langacker (e.g. 1991) and they also draw from the work of Talmy Givon (1995) and John Haiman $(1985,1998)$, who have argued for the 'iconicity' of syntax and who have sought to show that the construction of sentences often follows patterns which map the patterns of the event sequences that the sentences are about.

3 At least so it seems. Since Hauser, Chomsky and Fitch (2002) this is less clear, for according to these authors, as just mentioned, the cognitive capacity that makes recursive syntax possible could derive from a general capacity that has to do with the organism interacting with its physical environment, although not one developed in relation to communication, necessarily. For Armstrong and Wilcox (p.55) this paper is "a positive step by Chomsky" although they are anxious to show that the cognitive capacities leading to recursion in language "does involve communication" and they believe they can show this by means of Stokoe's notion of 'semantic phonology'. 
This framework is not, of course, the framework that has been the main guide in the development of linguistics, at least not in North America, during the central fifty years of the twentieth century. According to this framework it is maintained as a cardinal principal that linguistic form is to be studied separately from meaning. This is a consequence of the doctrine of the arbitrariness of the linguistic sign which came to be firmly established as an axiom of modern linguistics at the beginning of the twentieth century, largely as a result of the influence of Saussure's Cours, a doctrine still widely adhered to today (for one - out of many useful discussions of this see Waugh 2000). The belief in the arbitrariness of the relation between a linguistic sign and its meaning led to the attempt to develop the analysis of language as a system of relationships between forms, without taking meaning into consideration. Accordingly, there is the study of the sounds of speech, or phonology, in which the function of speech sounds is studied from the point of view of how they serve to keep meaning-bearing units apart; of morphology in which the principles according to which meaning-bearing units are constructed out of the speech-sound units that are distinguished in phonology are sought out; and syntax, in which the principles governing the way morphological units are organised together into sentences are analysed. Meaning is to be studied separately as semantics. In the strict structuralist paradigm, the main concern is with how units of meaning, however these are to be defined, are themselves patterned in relation to the language's morphology and grammar. The issue of how forms and meanings are related has largely been avoided.

This approach to the analysis of language was especially dominant at the time William Stokoe undertook his pioneering analyses of American Sign Language (he acquired his knowledge of linguistics under the guidance of George Trager and Henry Lee Smith at the University of Buffalo, who were among the most prominent exponents of this 'structural' approach to language - see Stokoe 1960: 3) and his analysis of sign language structure represented an attempt to establish that something analogous to phonology and morphology could be shown to be present in the sign language he studied. Subsequent developments in sign language linguistics have, until recently, largely followed this model, even though those features of signed language that derive from the fact that it is a language constructed of visible bodily actions that can exploit space as a medium for significant linguistic contrast cannot easily be accommodated.

The motivation for doing this is to be understood, at least in part, against the background of a prejudice against sign language that had long been prevalent. As Armstrong and Wilcox describe it, this stems from the development of a movement that led to the rejection of sign language as a 'language' in the formal sense. Although, from the time of Abbé de l'Epée in Paris in the 18th Century, who was among the first to explicitly recognise the linguistic value of the visual communication systems used by the deaf, there were many who promoted sign language as a medium of education for the deaf, there were many others who were opposed to this. The advocates of an oral approach to deaf education argued that signing was mere pantomime, it was capable only of concrete expression, and it would be incapable of serving as a vehicle for abstract thought and reasoning. To use it as a medium for the education of the deaf would forever condemn them to a second class social and mental life. This view gained wide official sanction after 
the Congress of Educators of the Deaf held in Milan in 1880, where a resolution that stated that the use of sign was to be banned in all instructional contexts and only methods which sought to teach the deaf to use spoken languages were to be followed (Facchini 1983). This had far reaching effects and led to the complete marginalisation of signing in schools for the deaf (Baynton 1996, 2002) and it also contributed to the great decline in interest in gesture languages of any sort, indeed in 'gesture' in general, that followed soon after this (see Kendon 2004: 62-83; Kendon 2007).

This dismissal of sign language and the corresponding lack of interest in it, persisted well into the twentieth century. Consequently William Stokoe's work was at first met with considerable opposition among the educators of the deaf and with scepticism in the academic community. ${ }^{4}$ However, Stokoe's own tenacity in promoting his view, which received important support from certain prominent linguistic scholars, ${ }^{5}$ eventually wore down this scepticism to the point that, within a few years of his first publication a number of linguistically trained students took up the serious study of signed language from a linguistic point of view. In the development of signed language linguistics that followed, carried out at first by a relatively small group of investigators, a great deal of what was done was motivated by a desire to show that signed languages were "just like" spoken languages in every important respect. If this could be shown, it would make them fully worthy as a medium for the expression of abstract thought and completely appropriate as a medium of education. There was the fear that if it was admitted that something like 'gesture' operated in signed language, the campaign to legitimise signed language as being fully the equivalent of spoken language would be undermined. This meant that the rather obvious and pervasive iconicity that is to be found in signed lexicons, phenomena such as the use of space to establish grammatical relations within sentences, the use of so-called 'classifiers', and other phenomena that seem to be suspiciously similar to the 'gesturing' observed among non-signers, have either been played down in importance or have been explained away with attempts to show that they serve abstract grammatical functions that are like those found in spoken language grammars and thus have nothing to do with 'gesture'. As Wilcox has put it in another publication, in this approach it is necessary to "bleach language of all its bodily substance until we arrive at the ultimate abstraction: disembodied language, pure structural relations" (Wilcox 2004:151).

Armstrong and Wilcox, on the contrary wish to show that the linguistic characteristics of signed languages, the features that qualify them to be referred to as

\footnotetext{
${ }^{4}$ See the review of Stokoe (1960) by Herbert Landar in Language in 1961 who concludes his review by wondering how many linguists will share Stokoe's conviction that "the communicative activity of persons using this language is truly linguistic", given that "a signalling system which does not involve a vocal-auditory channel directly connecting addresser and addressee lacks a crucial design feature of language". (Lander 1961: 271).

${ }^{5}$ Notably William Austin at Georgetown University as well as Trager and Smith, who had promoted Stokoe's first publication. Stokoe addressed the Georgetown Roundtable in Linguistics in 1966. Thomas A. Sebeok also played an important role. He it was that obtained the necessary support for starting up the journal Sign Language Studies, first published by Mouton of the Hague with the support of the Indiana University Research Center for the Language Sciences, of which Sebeok was the director.
} 
'language' in what I referred to above as the formal sense, are emergent properties of processes that serve to transform expressive actions that do not have these characteristics (often referred to as 'gestures') into forms that do. In the context of contemporary sign language linguistics this can still appear somewhat radical, however a point of view compatible with that being developed in this book by Armstrong and Wilcox is now becoming more widespread, as may be seen in the work of Bouvet (1997), Cuxac (see Cuxac and Sallandre 2007), Taub (2001), Liddell (2003), Russo (2004), among others.

These considerations make plausible the idea that a language can be created out of modes of action that re-present in pantomimic, depictive and diagrammatic form, event descriptions and references to objects and ideas, and that we can see how such modes of action might come to be used as a way of communicating to others memories, perceptions and thoughts. Through processes of this sort we can imagine how a shared conventional system might come into being, that would have all the marks of a 'language' in the 'formal' sense.

\section{The relevance of palaeontology, neurophysiology and ontogenesis}

However, to support the view that these processes of language emergence are also the historical processes by which present day languages came into being, other kinds of considerations must be brought to bear. Since we cannot travel back in time to see what humans or their predecessors were like before they had language, we have to depend upon various kinds of information that can make a backwards reconstruction possible. Today, for this kind of backwards reconstruction, it is common to rely upon findings from the comparative study of the behaviour of living primates, recent work in the neurophysiology of speech, vocalisation, perception and action in both humans and in other primates, observations on how language is acquired in the course of growing up, and the palaeontology of the hominidae.

Armstrong and Wilcox summarize some of these findings in Chapter 2 of their book. Thus, it has been argued that human spoken language cannot easily be derived from the systems of vocalisations in our primate relatives, such as chimpanzees and bonobos, insofar as the neural systems by which these are controlled are quite different from those found in humans, who have full voluntary control over their vocalisations, whereas apes and monkeys do not (Ploog 2002 provides a recent summary). As the 'ape language' experiments of the Gardners, Premak and the Rumbaughs have shown, apes such as chimpanzees and bonobos can master symbolic expression to some degree, and, as the Gardners showed, they can be taught to express themselves symbolically through visible manual actions, but they cannot be taught to speak. ${ }^{6}$ Work on the neurophysiology of human speech perception and production and the perception and production of manu-

6 It was this discovery that played an important role in prompting Hewes' first formulations of modern versions of the 'gestural origins' theory (Hewes 1973). It also played a not unimportant role in providing a 'push' for the development of a modern linguistics of signed language (see Kendon 2002a). 
al actions, including the manual actions of signing, suggests that the centres in the brain controlling these processes overlap anatomically and are functionally co-involved (see Kimura 1993). Much prominence has been given to the discoveries of Rizzolati and his colleagues of so-called 'mirror neurons' which suggest a neurophysiological basis for the process by which monkeys and people understand each other's actions. It has been claimed by Rizzolati and Arbib (1998) that the area of the cortex of the rhesus monkey where mirror neurons were first observed is homologous to the area of the human cortex known as Broca's area, which is known to be much involved in spoken language production. Armstrong and Wilcox note that, if substantiated, this "would suggest specifically that the brain region in humans that provides the sine qua non for speech may have started out subserving instrumental manual activities that became gestural and communicative." (p. 26). Studies in the emergence of language in the course of human development (which Armstrong and Wilcox refer to but briefly, however) show that the first forms of action that have a referential function to be observed are gestural and that, at a certain stage, infants may use a mixed media vocabulary, some items being gestural, others being vocal, although beyond the age of two years hearing children shift definitively in the direction of a spoken vocabulary (Capirci, et al. 2002 provides an overview). As for the palaeontological evidence, it is known that bipedalism in the line leading to humans emerged very early and that this was associated with a development of the anatomy of the hand which led it to become much like a modern hand, well before the changes in the upper respiratory and vocal tracts took place that seem to allow for the production of modern speech. It is suggested that bipedalism would have freed the hands for functions other than locomotion, including their use in expression. Armstrong and Wilcox end their discussion of this range of findings by saying "from this evidence it seems reasonable to conclude that the earliest language-like behavior of the hominids involved visible, especially iconic and indexic manual signs...' (p. 30).

\section{The problem of speech}

Several different lines of evidence, then, can be added up to support the hypothesis that the first step in the evolution towards linguistic expression was taken with the employment of visible action, or gesture, for referential expression. Yet, as has often been pointed out, this seemingly attractive hypothesis faces, as MacNeilage (1998: 232) has put it, an insuperable problem. Languages are overwhelmingly spoken. Furthermore, humans appear to be specialised anatomically to be speaking animals (Lenneberg 1967, Lieberman 2006). If language first emerged as visible gesture it seems puzzling that speaking is its specialised and preferred modality.

Armstrong and Wilcox, although they recognise this problem, offer little that is very convincing in their attempts to overcome it. First of all they argue that there is no fundamental difference between 'speech' and 'sign'. They do this by exploiting two quite different ways in which the word 'gesture' has been used. This allows them to argue that speech is, after all, just a form of gesture. Most commonly, the word 'gesture' is used to refer to visible bodily actions that are 
expressive, and the term is usually applied to expressive actions that are deemed to be more or less voluntary (see Kendon 2004: 7-16 for some discussion of this usage; see also the Oxford English Dictionary, Second Edition 1989). The authors use 'gesture' in this sense, much of the time. However, on p. 33 they adopt a definition of the word that they take from Studdert-Kennedy (1987, p. 77), an advocate of the articulatory approach to phonetics, that "a gesture is a functional unit, an equivalence class of coordinated movements that achieve some end". ${ }^{7}$ In the light of this they claim that the issue of a transition from gesture to speech in the history of language evolution is a false problem. They write: “... there was in fact no Great Shift from gesture to speech. ... The difference between visible gestures and speech sounds is not that one is gestural and the other is not - they are both gestural in the sense that both depend upon planned sequences of musculo-skeletal actions" (p. 67). Putting it this way, that is, to say that 'gesture' and 'speech' are the same because they are both accomplished by "planned sequences of musculo-skeletal actions" is merely to utter a truism. It does not provide an answer to the problem. You might as well say that gesture and speech are no different from cutting down trees, stroking cats, driving cars or eating meat with a knife and fork. Such activities are also accomplished by "planned sequences of musculo-skeletal actions". The claim that 'gesture' and 'speech' are the same also completely glosses over the differences in the perceptual processes that must be involved in the comprehension of 'gesture' on the one hand, which involves visual perception, and 'speech', on the other, which involves auditory perception.

Nevertheless, with this attempt to resolve the problem of how a language of gesture might have become a spoken language, no matter how poorly it has been expressed, an important point is being made. This is, that 'gesture' and 'speech' are both voluntary forms of action undertaken with what might be referred to as 'semantic intent'. Later, we will make reference to MacNeilage's (2008) ideas on the origin of speech, according to which the actions of speech are derived from the manipulatory actions of the mouth in the processing and ingestion of food. 'Gestures' (in the more usual sense of the word) may also be derived from manipulatory actions which, furthermore, may be closely coordinated with mouth actions, since both mouth and hand are involved together in the activities of food getting and ingestion. In primates, hands are used to transport food to the mouth, hence close coordination between hand action and mouth action is required. As will be mentioned again later, there is evidence for close relationships between the neurological systems that control the voluntary actions of the hand and those of the mouth. Perhaps mouth actions and hand actions acquired semantic uses

\footnotetext{
7 In the passage from which these words have been taken, Studdert-Kennedy is not providing a general definition of word 'gesture' but is clarifying an implication of using this word to refer to motions of the tongue and lips in the production of speech. He argues that an approach that examines speech development from the point of view of how the child comes to be able to make the movements of tongue and lips, etc., that are necessary for speech production, will throw more light on these processes than an acoustic approach. He uses the word gesture in this context in the way that is customary among articulatory phoneticians, a use that is quite specialised and has nothing to do with the use of the term as a way of referring to expressive visible action. Just because articulatory phoneticians have adopted the term 'gesture' to refer to the articulatory actions of speaking is no grounds at all for claiming that speech is 'gesture' and thus not really different from what speakers, or signers, for that matter, do with their hands when they speak!
} 
jointly. At some level, they can be regarded as components of a single system. This may be the point that Armstrong and Wilcox are trying to make.

It will be clear that the problem of the transition from 'gesture' to 'speech' which a gesture theory of language origins will have to face will be much greater if one takes the position, as Corballis does, that languages much like modern deaf sign languages were elaborated before Homo began to use speech. Although, as we have noted, Armstrong and Wilcox do claim that signed languages were the original form of language, they are not consistent in this view. They suggest that their theory of language origins "does not require a transition from a period in which human ancestors used only visible gestures to one in which modern humans use only acoustic gestures" (p. 37). They continue: "At no time in our evolutionary history did communication take place in a single modality" and they go on to remind us of the work of McNeill (1992), among others, that makes it "quite clear that humans gesture while they vocalize (p. 37)." This is, of course, a matter of common observation. McNeill is worthy of mention here, however, not as an 'authority' who confirms an obvious fact but because, in his work, he has shown that gesture and speech are co-produced as if, that is to say, they are "two aspects of the same process of utterance" (Kendon 1980). Hence gestural expression and spoken language expression are related at some very deep level.

Armstrong and Wilcox thus recognise that the multimodality of linguistic expression they propose for our ancestors has persisted among us to this day. They suggest, however, that in the course of evolutionary history, there has been a shift in the "relative informational load carried by visible versus audible gestures" ( $p$. 37). They speculate that this shift might have come about as tasks such as foodgathering and food-processing and tool-making, which must be transmitted to the young, become ever more complex to explain. Here they refer to an idea put forward by Barbara King (1994) that processes of "information donation" from old to young would be selected for among primates, as changes in the kinds of environments exploited were linked to an increase in variability and flexibility in food getting strategies, as well as the use of tools. If language is mostly carried out in visible gesture, this will interfere with manual tasks. If the informational burden shifts to the vocal channel, however, the hands become free to engage in manual tasks while, at the same time, what is being done can be explained to the young. 8

They also add some of the other reasons that are usually given as to why speech was selected for, rather than gesture, such as the greater energy efficiency of speech, the fact that with speech one can communicate in the dark or from behind rocks or round corners, and the like. As MacNeilage (1998: 232) has pointed out, however, if these reasons can explain why we have speech, they could also be adduced to argue that we would never have begun with gesture as a modality for language in the first place. The postulation of a gestural language as a stage that precedes spoken language seems, thus, superfluous (see also Bradshaw 2003). Modern sign languages (produced, as they are, by modern humans with all the benefits of human evolutionary history behind them) can be understood as latter

\footnotetext{
${ }^{8}$ A similar idea has been suggested by Corballis. He thinks that the great late Pleistocene "explosion" coincided with a shift to spoken language. All those cultural productions now became possible because the hands were freed, at last, from the burdens of signing.
} 
day adaptations. They reflect the ingenuity and flexibility of the language faculty, but they tell us nothing about the origins of that faculty.

I find it hard to see how this last point can be refuted. However, perhaps the main claim that Armstrong and Wilcox are making is that, although modern sign languages cannot tell us about the origins of the language faculty, they can tell us a great deal about the nature of that faculty. They can make clear for us the processes by which languages form, not in historical terms, but in terms of the cognitive capacities and processes that are necessary if human languages are to come into being at all and which are continuously and currently involved in the processes by which languages are acquired and maintained in modern humans. Armstrong and Wilcox suggest that if we can see how pictures become graphic units in a writing system or how depictive gestures and pantomimes become signs, if we can see the rules of syntax emerging through a process of ritualisation and abstraction from mimetic representations of events in visible gesture, the implication is that the development of vocal lexical items and their syntactical organisation must follow a similar course. Spoken language, too, must arise from forms of vocal action that are not, in the first place, 'language'. Indeed, Armstrong and Wilcox assert that "the processes at work in the elaboration of signed languages are analogous to what occurred and continues to occur in the evolution of speech" (p.31).

\section{Sound symbolism}

Surprisingly, they say very little in support of this claim. The best they offer in their account of how speech came about as a vehicle for language is to suppose that by some process of association vocalisations that co-occurred with gestures gradually came to stand for the things the gestures referred to. This, combined with the fact that using speech is so much more convenient in many ways (as noted above) is offered as a sufficient explanation for the establishment of spoken language and its modern predominance. Yet, if they are right, we ought to be able to see that the forms found in spoken languages show evidence of being emergent products of the same processes that the authors show to govern the emergence of signed languages and writing.

I suggest that, in fact, there is plenty of evidence that supports this claim. This may be found in the cluster of phenomena in spoken language that is often (and somewhat misleadingly) referred to as "sound symbolism". That is, the cluster of phenomena in spoken language that shows that, in many different ways, there is often a relationship between the phonological forms of speech units and their meanings. Although the phenomena of "sound symbolism" are commonly downplayed by many linguists, or looked upon as being only of marginal interest, there is evidence that suggests that the "sound shapes" of language (Jakobson and Waugh 1979) often are not unrelated to meaning and, in consequence, may play a role in how spoken language functions that should not be ignored. As Hinton, Nichols and Ohala (1994:1) observe "sound symbolism plays a considerably larger role in language than scholarship has hitherto recognized". Janis Nuckolls (1999) notes that a 'paradigm shift' is underway. As she has written elsewhere (Nuckolls 1996), referring to her work on Pasteza Quechua, but this seems to me to have an entirely general application, "Sound...is a modality for representing the natural- 
ness or unnaturalness of perceptual experience. The movements of the mouth, the shaping of the vocal tract, the fluctuating pitch of the voice are all uses of the body to imitate movements and processes of perceptual experience." (p. 5). Putting the voice and vocal tract to work in the service of such representations of movements and perceptual experience appears to be fundamental to the emergence of spoken languages.

The literature on "sound symbolism", or "phonosemantics" as it might be better to call it, is diverse and vast (see, for example, Wescott 1971, Jakobson and Waugh 1979, Nuckolls 1999, Hinton et al. 1994. For an historical discussion see Genette 1995). Despite the widespread adherence to the doctrine of the arbitrariness of the linguistic sign, there has persisted in linguistics a strong countercurrent that asserts that, indeed, meaning and the sounds of speech shape each other. This countercurrent has found expression in a number of the important voices of modern linguistics, including Sapir (1929), Jespersen (1922), Jakobson (1971), Bolinger (see papers reprinted in Bolinger 1965), among others. Notwithstanding, no coherent framework has yet emerged which makes it possible to see how the phenomena of phonosemantics can be integrated with structuralist accounts of language. Possibly it cannot be fully integrated and it may be that we will have to conclude, as Nuckolls suggests, that "languages...[are] essentially heterogeneous systems in which meanings are conveyed using a combination of elements...". However, there does seem to be a persistent set of forces that pull the sets of actions we use for linguistic expression toward a kind of systematicity and, as they do so, they tend to override the "imitation of movements and perceptual experience" and obscure the expressivity which, however, never stops welling up from the efforts of individuals to make themselves understood, to make themselves enjoyed as entertaining foci of attention, and to present their perceptions, thoughts and experiences in ways that are, for others, vivid and involving. These efforts, ultimately, do not take as their starting point the formal rules of phonology and syntax. Givón is surely right when he suggests that "iconicity [is] the truly general case in the coding, representation and communication of experience, and symbols a mere extreme case on the iconic scale." (Givón 1985: 214). Modes of expression, insofar as they establish themselves as socially shared systems, tend toward a systematicity that schematises, abbreviates, and regularises, but this never completely obscures the "iconicity" which is almost always its starting point. Armstrong and Wilcox have shown this clearly for systems that use the kinesic modality. When we look in the right way, however, we can also see this in the modality of speech.

Perhaps the most widely attested feature of spoken language which shows a motivated link between sound and meaning is the phenomenon of "ideophones", sometimes also referred to as "expressives", "onomatopoetics" or "mimetics." This class of verbal item was first described by Doke (1935) for Bantu languages of Africa, and he it was who first called them 'ideophones'. However ideophones have since been described and shown to be widespread in a large number of different languages throughout the world, including Australian languages, South Indian languages, Korean, Japanese, several languages of South America and also of North America (see Voeltz and Kilian-Hatz 2001). They are less common in Western European languages, although English, for example, can certainly be re- 
garded as making use of them, as in 'sound words' such as "boo", "bah", "bam”, "wham" and so forth. In English, however, as also, for example, in Swedish, such expressions tend to be employed mainly in whimsical, playful or childish speech or in the speech of adolescents (Nordberg 1986), whereas in the many other languages mentioned in which they have been described they may play a common role in everyday discourse and even in formal discourse.

In many languages, although not in all, ideophones have special phonological features and they often are isolated syntactically within the sentences in which they occur. Semantically, they often function rather like adverbs. Their origins perhaps may be understood in terms of the processes of physiognomic perception in which similarities between referent and sign vehicle are actively constructed. Their usage is not always and only to express concrete experience or concrete aspects of manner of action, however. As Nuckolls (1996) has shown for Quechua they can serve in the expression of grammatical aspect such as completiveness. Although they have been widely described, there is as yet no generally accepted theoretical framework by which they can be incorporated into linguistic theory and many linguists regard them as standing outside the rest of language. Diffloth (1994: 108), for example, writing of what he calls 'expressives' in a Vietnamese language, suggests that they "constitute a parallel sublanguage grafted on and parasitic on, the conventional one."

However they may be regarded, ideophones provide an excellent demonstration of how vocal expressions can be constructed in a highly iconic fashion and yet be used as an integral part of normal spoken discourse. It is notable that in a number of languages it can be shown that many parts of speech, including verbs, adverbs and nouns are derived from ideophones (see, for example, McGregor 2001), attesting to a process in spoken language of 'ritualisation' similar to the process referred to by Armstrong and Wilcox by which lexical items in a signed language can often be shown to be derived from more directly analogue forms of visible bodily expression.

In almost all the languages that have been described, phonosemantic phenomena in phonology, morphology and syntax of one sort or another have been attested (Ciccotosto 1991 provides thorough survey). Various attempts have been made to identify the different kinds of phonosemantic phenomena. Hinton, Nichols and Ohala's (1994) classification provides a useful guide. Imitative sound symbolism or onomatapoeia includes the many groups of words that represent sounds that occur in the environment - in English we have such words as "crack", "bang", "swish", "tap", "knock", and the like. All languages have forms of this sort but since they are assimilated to each language's phonology, or follow different conventions, similar kinds of sounds may be represented onomatapoetically in different ways. It is nevertheless clear that sound imitation is a widespread mode of word-formation. Synesthetic sound symbolism refers to cases in which phenomena that are not acoustic receive a correlative acoustic representation. The most widely attested evidence for this is in so-called "magnitude symbolism" in which words for small things tend to have high front vowels (tiny, petit, piccolo) where words for large things have low back vowels (huge, grand, etc.) Although many exceptions can be adduced, this kind of magnitude sound-symbolism appears very widespread in the world's languages (Ultan 1978). Finally, there is so-called 
conventional sound symbolism in which certain phonemes or clusters of phonemes come to be associated in groups of words which share a common semantic theme. These are sometimes referred to as 'phonesthemes', a term first introduced by J. L. Firth (1930). In English we find, for example, words that begin with gl- cluster around the theme of light, as in glow, glimmer, gleam, glisten, glimpse. Likewise, there are other groups of words ending in -sh that cluster around the theme of a process of object transformation through violent action in which the object is transformed into small pieces or loses its shape, as in bash, crash, crush, squash, squish, and the like. As Waugh (2000) suggests, this is a widespread feature of English (see also Magnus 1999 and her Dictionary of English Sound accessible at http:// www.trismegistos.com/) but it is also widely found in other languages (for a thorough description of it in Indonesian see McCune 1983).

At the morphological level, various kinds of iconicity have been described. Reduplication, for example as a form of pluralisation, is a very widespread device. In English it tends to be used to express the idea of repetitive or reciprocal actions of various kinds, and in such cases reduplication is partial, since there is a vowel change from one part to the next, as in flim-flam, zig-zag, flip-flop, dilly-dally, see-saw, and the like. As has been pointed out by Wescott (1971), among others, plural forms are often longer than singular forms (ox vs. oxen, cat vs. cats, to give English examples), and similarly, intensification of adverbs or adjectives is often accomplished by increasing the number of syllables (as in Italian we have lungo, lunghissimo, caro, carissimo, etc.). At the syntactic level, as has been extensively demonstrated by Haiman (1985), among others, there is much evidence for the widespread use of a diagrammatic iconicity.

Phenomena that support the idea that there are systematic relations between the form of a linguistic expression and its meaning, are thus very widespread. The relations uncovered can be characterised in terms of a number of different 'iconic devices', as we have seen, which include sound imitation, synesthetic relations, and relationships that can be described as 'diagrammatic' (as in the case of reduplication or word lengthening for pluralisation, or syllabic doubling with vowel alternation that express repeated alternating actions). That this is so makes it clear that processes akin to what Werner referred to as 'physiognomic' processes are foundational in the creation of spoken language. As we have seen, Armstrong and Wilcox show that they are also foundational in sign language creation. It needs to be said, however, that if it is accepted that what lies at the start of language creation, in whatever modality, is the capacity to create referential actions following iconic principles, this is, of course, not a claim that languages in their contemporary function are no different from imitation and pantomime. The 'iconic hypothesis' might account for how language begins and it may account for an important component of the way in which languages continue to function, change and grow, but there are obviously many other factors at work as well.

\section{$7 \quad$ Iconicity in language: signed and spoken}

Armstrong and Wilcox, in their discussion of iconicity in sign language point out that a sign that is iconic is not therefore also not symbolic, that is, it is not therefore also not conventional. This is a point that Peirce himself recognised and has 
received modern discussion in Eco (1976). We can, in fact, set up a scale in terms of which signs may be said to be more or less conventional. This is as true in spoken signs as it is of gestured ones or graphic ones (compare the 'wild-tame' scale for sound words suggested by Rhodes 1994). As Diffloth (1994: 113) has written in reference to his analysis of expressives in Bahar (a language of Vietnam) in which he finds that high front vowels tend to be associated with expressing largeness while low back vowels tend to be associated with expressions of smallness (the reverse of the usual pattern), "two languages may easily use the same phonetic variable (vowel height) to convey the same range of sensations (size), and come up with exactly opposite solutions, both being equally iconic; all they need to do is focus upon different parts of the rich sensation package provided by articulatory gestures, in our case the volume of the tongue instead of the size of the air passage between it and the palate. Iconicity can be both physiologically motivated and culturally relative at the same time." This is exactly analogous to what is found in signed languages. An example often used to illustrate this is the comparison of the sign for 'tree' in American Sign Language, Danish Sign Language and Chinese Sign Language (see Klima and Bellugi 1979: 21). In ASL an erect forearm with a spread hand is used (depicting a vertical trunk with spreading branches at the top), in DSL the two hands together outline a sphere with a strait narrow object below it, in CSL the two hands posed with thumb and forefinger abducted and facing one another move upward, as if moving upwards along a vertical cylindrical object. In all three SLs certain features of a tree are abstracted, but different features are chosen in each case and a different mode of representation of these features is employed in each case. All three signs are 'iconic' since there is a relationship of resemblance between the actions of the sign and the features referred to in each case. At the same time all three signs are conventional. In Peircean terms, they are at once icons and symbols. Numerous other examples can be found, drawing on many different sign languages, that illustrate the same point.

As Armstrong and Wilcox explain, there has been, in sign language linguistics, a great reluctance to admit the importance of the role iconicity in sign language because most sign language linguists (and others) have adopted the dogma that for a sign to be truly linguistic it must be arbitrary. Because students of sign language since Stokoe's pioneering work have been anxious to demonstrate the true linguistic status of signed language they have been afraid to admit the role of iconicity because to do so, they feel, would undermine its linguistic status and throw it back to a view of it as "mere gesture", and therefore unworthy of being a serious medium of expression, instruction and thought. The work of Armstrong and Wilcox, among others, is contributing to a shift in this attitude, and, as we have already mentioned, a number of sign language linguists such as Sarah Taub, Christian Cuxac and Scott Liddell, among others, are now recognising the central importance of iconicity in signed languages. Oddly enough, spoken language linguists are likewise nervous about iconicity in spoken language, although for different reasons. The caution with which they treat the topic of phonosemantics, for example, is based on the way in which it is apparently in conflict with the principle that sounds function in language solely to keep linguistic units apart and do not serve to convey meaning in themselves. As Jespersen (1922) has also pointed out, the willingness of most linguists to dismiss iconicity as a princi- 
ple of any importance in spoken language derived also from de Saussure's argument that words in current speech that seem to be phonically iconic can often be shown by their etymological history to be descended from words that do not seem to have such iconicity. Jespersen comments, however, that "modern linguistic science...is so preoccupied...with the origin of words, that it pays much more attention to what words have come from than to what they have come to be. If a word has not always been suggestive on account of its sound, then its actual suggestiveness is left out of account and may even be declared to be merely faniciful." (p.410). Jespersen demonstrates in his chapter on Sound Symbolism however, that the sound suggestiveness of words, whatever their etymologies may be, can play an important role in how they are used, how they relate to one another within the language contemporaneously, and what sort of survival history they may have within a given speech community. Bolinger, in several papers (reprinted in Bolinger 1965), provides a further exploration of this theme. As we have seen, recent and current work, especially anthropological linguistic work which is being done on many different languages in many different parts of the world, both supports and amplifies Jespersen's observations. It seems that a way of thinking about language is needed in which both the creative force of iconic representation (at every level) is acknowledged as well as the tendency toward systematicity that often overrides such representation. This tendency, also ever present, of course, derives from the nature of language as a social institution. As Nuckolls (1999: 246 ) concludes, the evidence points toward a "view of language as a system structured by competing tendencies." This is certainly the view that Armstrong and Wilcox provide us with for signed languages and it seems clear from the work in spoken language linguistics we have just been referring to that these can be viewed in just the same way.

Such considerations might suggest, thus, that in both the medium of visible action and in the medium of vocalisation, similar representational processes of a mimetic or iconic type are in operation. Armstrong and Wilcox indeed suggest this and they claim, on the basis of this, that spoken languages and signed languages form a unity. However, there is a further aspect that can be brought in which further strengthens the idea that there is a unity between signed and spoken language, and that is that actions of the mouth and manual actions enter into some kind of synergistic relationship with one another.

\section{Synergies of hand and mouth and the 'tongue gesture' hypothesis}

Armstrong and Wilcox do make some reference to work that suggests that neural control systems involving the mouth actions of speech and those involving actions of the forelimbs perhaps have features in common. They do not mention, however, the recent work of Gentilucci and colleagues in which mouth action and hand action synergies have been demonstrated. In a series of experiments (see Gentilucci and Dalla Volta 2007 for a review), Gentilucci and his co-workers have reported observations that suggest that forms of action made by the mouth can be paralleled by forms of hand action. Thus if a person is asked to simultaneously pick up a small object (such as a cherry) and pronounce a syllable such as 'Ba' 
as he does so, he shows a tendency to use a smaller mouth opening than if the simultaneous hand action is to grasp a large object, such as an apple. And, by the same token, simultaneous grasping actions of the hand may be smaller if the syllable to be pronounced is 'Bi' rather than 'Ba'. Such observations seem to suggest that there is a kind of co-ordination in the control system that govern hand and mouth actions. Gentilucci and colleagues have speculated that this kind of handmouth co-ordination came about in evolution as a consequence of the use of the hands to transport food to the mouth. They suggest it may be phylogenetically very old, perhaps having its origins from the time when primate ancestors began to live in trees in a three dimensional manner. Using hind-limbs and one forelimb to hold on to, the other forelimb could be freed to reach for and grasp fruit or other food items, which would then be transported to the mouth by the hand. Such a mode of food getting could facilitate the development of hand-mouth coordination control systems and, so it is suggested, it is in this that lies the origin of the neuro-motor mouth-forelimb synergies that various lines of observation suggest, including those of Gentilucci and his colleagues.

In such an approach we may see the origins of what makes possible a kind of paralleling of mouth and hand action. Indeed, it is in this that we might have an idea about how expressive actions in visible action systems such as the forelimbs could have come to be transferred to actions of the mouth. In supposing that, in speech, we can see in operation processes that transform mimetic or iconic expressions into systematised and schematised forms of action that have 'linguistic' features, as several writers have pointed out, this need by no means needs to be confined to sound imitations only. Undoubtedly onomatopoeia has played and continues to play a role in word formation processes, as the survey above shows, but, as Paget (1930) pointed out a long time ago, motions of the vocal articulatory apparatus can also be made that parallel the actions in space of the hands. If there is vocalisation during actions of this sort, the aural consequences will differentiate accordingly and the patterns of sound resulting, though not like patterns of sound such as the cry of animals and the like, are nevertheless consistently correlated with changes in the geometry of the vocal tract. It is in terms of this idea, for example, that one might account for the origins of the association found in many languages between high front vowels and smallness of size. Several scholars have, in the past, sought to trace in mouth actions mimic representations of various kinds. Before Paget made this suggestion, in 1862 John Rae had proposed it in relation to material he had assembled for a Polynesian language (Rae's monograph on this was published as an appendix to Paget's book). The idea had also been proposed by Alfred Russell Wallace (1895). Scholars of language who have pursued the same idea include Johannesson (1952), who proposed the tongue gesture theory for language origins on the basis of an analysis of Indo-European roots. A somewhat different approach was followed by Mary LeCron Foster (see, for example, Foster 1992,1999). Some additional support for this idea may also be found in Bencie Woll's (2001) suggestion that what she has termed 'echo phonology' in sign language, in which a manual sign is combined with an action of the mouth which has dynamic features that have something in common with the hand actions of the sign, could provide an example of how a spoken form can be derived "quite naturally" from a gestural form. 
However, even if the 'tongue-gesture' idea were to be accepted as a plausible way of accounting for how mouth actions got involved in the production of referential gestures and in this way allowed for the preservation of the idea of the "gestural origins of language' when the original 'gestures' were made both as visible actions and as mouth actions - these mouth actions having aural consequences in the way they modified the concurrent vocal output (in this way, thus, avoiding the problem that a gesture-language-first scenario poses), we still need to add something that allows us to understand how the actions of the mouth and associated vocalisation came to be available, as it were, so that they could be recruited into the referential gesture function. For this to be possible an elaborate and voluntary control of the vocal system must already have been in place. In other words, a scenario for the evolution of the human speech apparatus and its neuro-motor control systems is also needed.

For this we may turn to MacNeilage (2008) who presents a scenario in which speech is seen as deriving from rhythmic open-closing mouth actions that have their original form in the mastication of food. As he reminds us, among primates extensive use is made of mouth and tongue actions in communication which are distinct from use of the mouth in the production of calls. Many species of monkeys and apes use mouth gestures such as lip-smacking, tongue protrusion or lip protrusion, some also engage in low volume "grunting", the acoustic properties of which may be modified with tongue actions. Already, widespread in various primate genera, thus, complex mouth actions are being used in communication, in forms of action that are under voluntary control. MacNeilage sees these as deriving from rhythmic mandible oscillations that ultimately originate in the action systems involved in mastication.

Speech, however, even if it is the dominant vehicle for language, must be regarded as but a component of language. As the existence of sign languages demonstrate, we can have languages that do not involve speech. A scenario for the evolution of speech, in particular, thus, will not be the same as a scenario for the evolution of other language components, such as a capacity for the production and understanding of symbolic actions.

\section{Common features of signed and spoken discourse}

This, then, is one way in which we can explore the issue of the unity of spoken language and signed language. There is another way to approach this question, however, and that is through comparisons with how signers and speakers construct their discourses. This is also something that Armstrong and Wilcox could have made more of in their discussion than they do.

If, in comparing signed discourse with spoken discourse we compare directly actual performed spoken discourse with signed discourse we can see how, in both speakers and signers, extensive and flexible use is made of a range of expressive resources, many of which are highly analogous to one another, if not actually the same (see, for example, Kendon 1993, 2004: 307-325 and see also Enfield 2004). For example, speakers employ certain kinds of well-established gestures to express certain kinds of grammatical functions, such as negation (Kendon 2002b, 2004: 248-264), interrogation of various kinds, topic-comment marking and focus 
marking (Kendon 1995, Kendon 2004: 225ff), they inflect their deictic gestures with different kinds of handshapes to mark the discourse status of the objects they are making reference to (Kendon and Versante 2003, Kendon 2004: 199-224; Wilkins 2003) and they can employ descriptive gestures as if to display objects of reference in their discourse and show certain properties of them in ways that appear to parallel the way in which so-called 'classifiers' are used in signed discourse (see Kendon 2004: 316-324). These various kinesic devices just referred to can be employed simultaneously with the unfolding of speech, they can serve to 'bracket' stretches of discourse as a way of indicating its status in much the same way that signers can, when they use facial actions or head actions to mark interrogatives, subordinate clauses, or negations (McClave 2001). Furthermore, speakers may employ their hands to mark out contrasting spaces to refer to different actors in a narration (Gullberg 1998; Kendon 2004: 310-315) or to establish different spaces for different components of an argument (compare the common expression "on the one hand...on the other hand"). As Calbris (1990) and Kendon (1993) have described, speakers use their hands to indicate different kinds of spaces and different kinds of progressions along virtual lines in space to indicate different time locations and movement through time, mapping out a temporal spatial layout which matches closely that observed in sign languages such as American Sign Language, French Sign Language or Italian Sign Language and British Sign Language). As I conclude in Kendon (1993): "The parallels between how space is used to express time in verbal metaphor, gesticulation and sign that we have illustrated suggest continuities between spoken language expressions, gesticulation and sign language, as if these modes of expression are all drawing on the same representational substrate." I suggest, accordingly, that symbolic representation by way of spoken language and symbolic representation by way of gesture, including sign, "appear less widely separated than might at first be thought. They may be regarded as different elaborations of a common underlying process" (p. b13).

Much more recently, sign language researchers have begun to confront in a systematic way the phenomenon of "simultaneity" - the way in which, in sign language discourse meanings are often built up with combinations of strands of simultaneously organised hand, face, mouth and head actions, of shifts in bodily stance and orientation (Vermeerbergen, Leeson and Crasborn 2007). The parallels with what speakers do, as is clear if we consider the detailed descriptions of gesture use in speakers to be found in Kendon (2004, see especially Chapters 8-13 and Chapter 15) as well as in the work of people like Isabella Poggi (Poggi 2007) make it clear that "simultaneity" is far from being a characteristic only of users of signed language. Speaker discourse performance also can involve a great deal of simultaneous expression. It is clear that a programme of collaborative and comparative work in which signed discourse and spoken discourse is directly compared as performances will show that there is very great overlap in how signers and speakers organise their linguistic expressions. As I conclude in Kendon (2004: 325): "As we speak or sign we constantly mix in with our discourse all manner of expressive devices, some more, some less well patterned. Signers use words and syntactic constructions, but they also modulate their performance of their signs in various ways, employ 'classifiers' and pull in kinesic expressions of all kinds, some from the kinesic vocabulary of the wider community, some improvised. Speakers act 
similarly. They use words and syntactic constructions, but they also use intonation patterns, voicings and vocalisations. And when they use gesture they reach out for strategies of expression that are also found in sign languages." If this view is correct it surely argues that 'sign' and 'gesture' and 'spoken expression' (lexical, prosodic, 'vocal gestural' and so forth) all spring from the same source. Sign languages may emerge only in particular circumstances, yet how they do so and the manner in which their users construct their utterances, follow principles of formation and performance that are of a piece with those used by speakers. Hence we see a unity in speech, sign and gesture and we see, furthermore, that they are interwoven together in a kind of complex tapestry.

\section{Conclusion}

Where does all this leave us with respect to the theory of the "gestural origin of language"? Armstrong and Wilcox have provided us with a concise up-to date summary, for the most part clearly written, that shows very well why the study of signed languages is making so important a contribution to our understanding of the capacities that are involved in the creation of language. They provide excellent support for the position outlined by Tylor (1865), for example, which claims that in the study of communication systems in the kinesic modality we can observe the processes by which communication systems come to acquire the cluster of features which leads us to attach the term 'language' to them. However, this should not be confused with an account of how 'language' actually emerged in the course of the history of the human species. All of the processes that Armstrong and Wilcox point to depend upon cognitive capacities that modern humans already have. To show how these cognitive capacities arose historically will require arguments and evidence different from those that have been provided in this book. Since such arguments and evidence can never be provided definitively, however, we may expect to see many more publications offering solutions to the problem of language origins.

\section{References}

Armstrong, David F. (1999). Orignal Signs: Gesture, Sign and the Sources of Language. Washington, D.C.: Gallaudet University Press.

Armstrong, David F., William C. Stokoe, and Sherman E. Wilcox (1995). Gesture and the Nature of Language. Cambridge: Cambridge University Press.

Armstrong, David F. and Sherman Wilcox (2007). The Gestural Origin of Language. New York: Oxford University Press

Baynton, Douglas C. (1996). Forbidden Signs: American Culture and the Campaign Against Sign Language. Chicago and London: University of Chicago Press.

Baynton, Douglas C. (2002). The Curious Death of Sign Language Studies in the Nineteenth Century. In David F. Armstrong, Michael A. Karchmer, and John Vickerey Van Cleve, eds. The Study of Signed Languages: Essays in Honor of William Stokoe. Washington, D. C.: Gallaudet University Press, 2002. 13-34.

Bellugi, Ursula, and Edward Klima, S. (1976). Two Faces of Sign: Iconic and Abstract. In Stevan R. Harnard, Horst D. Steklis, and Jane Lancaster, eds. Origins and Evolution of 
Language and Speech. Annals of the New York Academy of Science, Volume 280. New York: New York Academy of Sciences, 514-38.

Bergin, Thomas Goddard, and Max Harold Fisch (1984). The New Science of Giambattista Vico. Unabridged Translation of the Third Edition (1744) With the Addition of "Practic of the New Science." Ithaca and London: Cornell University Press.

Bickerton, Derek (1981). The Roots of Language. Ann Arbor: Karoma Publishers.

Bolinger , Dwight (1965). Forms of English: Accent, Morpheme, Order. Harvard: Harvard University Press.

Bouvet, Danielle (1997). Le Corps Et La Métaphre Dans Les Langues Gestuelles: A La Recherche Des Modes De Production Des Signes. Paris: L'Harmattan.

Bradshaw, John L. (2003). Gesture in Language Evolution: Could I But Raise My Hand to it! Brain and Behavioural Sciences, 26.2 (2003): 213-214.

Bråten, Stein, ed. (2007). On Being Moved: From Mirror Neurons to Empathy. Amsterdam and Philadelphia: John Benjamins.

Bulwer, John (1974 [1644]). Chirologia Or the Natural Language of the Hand, Etc. [And] Chironomia Or the Art of Manual Rhetoric, Etc. Edited with an Introduction by James W. Cleary. Carbondale and Edwardville, Illinois: Southern Illinois University Press.

Burling, Robbins (2005). The Talking Ape: How Language Evolved. Oxford: Oxford University Press.

Calbris, Geneviève (1990). Semiotics of French Gesture. Bloomington: Indiana University Press.

Capirci, Olga, M. Cristina Caselli, Jana Iverson and Elena Pizzuto (2002). Gesture and the Nature of Language in Infancy: The Role of Gesture as a Transitional Device En Route to Two-Word Speech. In David F. Armstrong, Michael A. Karchmer, and John Vickerey Van Cleve, eds. The Study of Signed Languages: Essays in Honor of William Stokoe. Washington, D. C.: Gallaudet University Press, 213-246.

Ciccotosto, Nick (1991). Sound Symbolism in Natural Languages. Docoral Dissertation, University of Florida

Corballis, Michael C. (2002) From Hand to Mouth: The Origins of Language. Princeton: Princeton University Press.

Cushing, Frank (1892). Manual Concepts. American Anthropologist. 5: 289-317.

Cuxac, Christian, and Marie-Anne Sallandre (2007). Iconicity and Arbitrariness in French Sign Language: Highly Iconic Structures, Degenerated Iconicity and Diagrammatic Iconicity. In Elena Pizzuto, Paola Pietandrea, and Raffael Simone, eds. Verbal and Signed Languages: Comparing Structures, Concepts and Methodologies. Berlin: Mouton de Gruyter, 13-33.

Damasio, Antonio and Kaspar Meyer (2008). Behind the loking glass. Nature, 454: 167-168.

Danesi, Marcel (1993). Vico, Metaphor and the Origin of Language. Bloomington: Indiana University Press.

Diffloth, Gérard (1984). i: big, a: small. In Leanne Hinton, Johanna Nichols and John J. Ohala, eds. Sound Symbolism. Cambridge: Cambridge University Press.

Doke, Clement Martin (1935). Bantu Linguistic Terminology. London: Longmans Green.

Eco, Umberto (1976). A Theory of Semiotics. Bloomington and London: Indiana University Press.

Enfield, N. J. (2004). On linear segmentation and combinatrorics in co-speech gesture: A symmetry dominance construciton in Lao fish trap descriptions. Semiotica, 142 (1/4): 57-123. 
Foster, Mary LeCron (1992). Body Process in the Evolution of Language. In Maxine SheetsJohnstone, ed. Giving the Body Its Due. Albany, N.Y.: State University of New York Press, 208-230.

Foster, Mary LeCron. (1999). The Reconstruction of the Evolution of Human Spoken Language. In Andrew Lock, and Charles R. Peters, eds. Handbook of Human Symbolic Evolution. Oxford: Blackwell Publishers, 747-775.

Frishberg, N. (1975). Arbitrariness and Iconicity: Historical Change in American Sign Language. Language. 51:696-719.

Genette, Gerard (1995). Mimologics. Translated by Thaïs E. Morgan. Lincoln and London: Univerity of Nebraska Press.

Gentilucci, Maurizio, and Riccardo Dalla Volta (2007). The Motor System and the Relationship Between Speech and Gesture. Gesture, 7 (2): 159-77.

Givon, Talmy (1985). Iconicity, isomorphism and non-arbitrarycoding in syntax. In John Haiman, ed. Iconicity in Syntax. Proceedings of a Symposium on Iconicity in Syntax, Stanford, June 24 -26, 1983. Amsterdam: John Benjamins.

Givon, Talmy (1995). Functionalism and Grammar, Amsterdam and London: John Benjamins.

Haiman, J. (1985) Natural Syntax: Iconicity and Erosion. Cambridge: Cambridge University Press.

Haiman, J. (1998). Talk is Cheap; Alienation, Sarcasm and the Evolution of Language. Oxford: Oxford University Press.

Hauser, Marc D., Noam Chomsky, and W. Tecumseh Fitch (2002). The Faculty of Language: What is it, Who Has it, and How Did it Evolve? Science, 298: 1569-1579.

Hewes, Gordon W. (1973). Primate Communication and the Gestural Origins of Language. Current Anthropology, 14: 5-24.

Hinton, Leanne, Johanna Nichols, and John J. Ohala, eds. Sound Symbolism. Cambridge: Cambridge University Press.

Hurford, James R. (2007). The Origins of Meaning. Oxford: Oxford University Press.

Jakobson, Roman (1966). Quest for the essence of language. Diogenes, 51: 21-37.

Jakobson, Roman, and Linda Waugh (1979). The Sound Shape of Language. Bloomington: Indiana University Press.

Jespersen, Otto (1922). Language: Its Nature, Development and Origin. New York: Henry Holt and Co.

Jóhannesson, Alexander (1952). Gestural Origin of Language. Evidence From Six "Unrelated" Languages. Rekjavík: H. F. Leiftur.

Kendon, Adam (1980). Gesticulation and speech:two aspects of the process of utterance. In Mary Ritchie Key, ed. The Relationship of Verbal and Nonverbal Communication. The Hague: Mouton, pp. 207-227.

Kendon, Adam (1991). Some Considerations for a Theory of Language Origins. Man (N.S.). 26: 602-619.

Kendon, Adam (1993). Space, Time and Gesture. Degrès, 74: 3a-16a.

Kendon, Adam (1995). Gestures as Illocutionary and Discourse Structure Markers in Southern Italian Conversation. Journal of Pragmatics, 23: 247-279.

Kendon, Adam. (2002a). Historical Observations on the Relationship Between Research on Sign Languages and Language Origins Theory. In David Armstrong, Michael A. Karchmar, and John Vickerey Van Cleve, eds. The Study of Signed Languages: Essays in Honor of William C. Stokoe. Washington, D. C.: Gallaudet University Press.

Kendon, Adam (2002b). Some uses of the head shake. Gesture, 2:147-183 
Kendon, Adam (2004). Gesture: Visible Action as Utterance. Cambridge: Cambridge University Press.

Kendon, Adam (2007). On the origins of modern gesture studies. In Susan Dunacn, Justine Cassell and Elena Levy, eds. Gesture and the Dynamic Dimension of Language. Amsterdam: John Benjamins, pp. 13-28.

Kendon, Adam, and Laura Versante (2002). Pointing By Hand in 'Neapolitan'. In Sotaro Kita, ed. Pointing: Where Language, Culture and Cognition Meet. Ed. Mahwah, New Jersey: Lawrence Erlbaum, 109-137.

Kimura, Doreen (1993). Neuromotor Mecahnisms in Human Communication. Oxford: Oxford University Press.

King, Barbara J. (1994). The Infomation Continuum: Evolution of Social Information Transfer in Monkeys, Apes and Hominids. Santa Fe, New Mexico: SAR Press.

Klima, Edward A., and Ursula Bellugi (1979). The Signs of Language. Cambridge, MA: Harvard University Press.

Lander, Herbert. (1961). Review of Sign Language Structure: An Outline of the Visual Communication Systems of the American Deaf By William C. Stokoe. Language, 37: 269-271.

Langacker, Ronald L. (1991). Concept, Image and Symbol: The Cognitive Basis of Grammar, Cognitive Linguistics Research, Vol. 1. Berlin: Mouton De Gruyter, 1991.

Lenneberg, Eric H. (1967). Biological Foundations of Language. New York and London: John Wiley and Sons.

Liddell, Scott K. (2003). Grammar, Gesture and Meaning in American Sign Language. Cambridge: Cambridge University Press.

Lieberman, Philip (2006). Toward an Evolutionary Biology of Language. Cambridge, MA: The Belknap Press of Harvard University Press.

Macneilage, Peter F. (1998). Evolution of the Mechanism of Language Output: Comparative Neurobiology of Vocal and Manual Communication. In James R. Hurford, M. Studdert-Kennedy, and Chris Knight, eds. Approaches to the Evolution of Language. Cambridge: Cambridge University Press, 222-240.

MacNeilage, Peter F. (2008). The Origin of Speech. Oxford: Oxford University Press.

McClave, Evelyn Zebrowski (2001). The Relationship Between Spontaneous Gestures of the Hearing and American Sign Language. Gesture, 1 (1): 51-72.

McNeill, David (1992). Hand and Mind. Chicago: Chicago University Press.

Nuckolls, Janis B. (1996). Sounds Like Life: Sound Symbolic Grammar, Performance and Cognition in Pastaza Quechua. Oxford: Oxford University Press.

Nuckolls, Janis B. (1999). The Case for Sound Symbolism. Annual Review of Anthropology, 28: 225-52.

Paget, R. A. S. (1978 [1930]). Human Speech. Some Observations, Experiments, and Conclusions as the to Nature, Origin, Purpose and Possible Improvement of Human Speech. New York: AMS Reprint 1978

Ploog, Deltev. (2002). Is the Neural Basis of Vocalization Different in Non-Human Primates and Homo Sapiens? In T. J. Crow, ed. The Speciation of Modern Homo Sapiens. Oxford: Oxford University Press, 121-35.

Poggi, Isabella (2007). Mind, Hand, Face and Body: A Goal and Belief View of Multimodal Communication. Berlin: Weidler Buchverlag.

Quintilianus, Marcus Fabius (1922). The Instituto Oratoria of Quintilian, With an English Translation By H. E. Butler. In Four Volumes. London; New York: William Heinemann; G. P. Putnam's and Sons. 
Rizzolatti, Giacomo, and Michael Arbib. (1998). Language Within Our Grasp. Trends in Neurosciences, 21: 188-94.

Rosenfeld, Sophia (2001). Language and Revolution in France: The Problem of Signs in Late Eighteenth Century France. Stanford, CA: Stanford University Press.

Russo, Tommaso. (2004). Iconicity and Productivity in Sign Language Discourse: An Analysis of Three LIS Discourse Registers. Sign Language Studies, 4(2): 164-97.

Sapir, Edward (1929). A study in phonetic symbolism. Journal of Experimental Psychology, 12:225-239.

Stamenov, Maxim I and Vittorio Gallese, eds. (2002). Mirror Neurons and the Evolution of Brain and Language. Amsterdam and Philadelphia: John Benjamins.

Stokoe, William C. (1960). Sign Language Structure: An Outline of the Visual Communication Systems of the American Deaf. Studies in Linguistics Place in Series: Occasional Papers No. 8. Buffalo, New York: Department of Anthropology and Linguistics, University of Buffalo.

Stokoe, William C. (1991). Semantic Phonology. Sign Language Studies, 71: 99-106.

Stokoe, William C.(2001). Language in Hand: Why Sign Came Before Speech. Washington, D. C.: Gallaudet University Press.

Studdert-Kennedy, M. (1987). The Phoneme as a Perceptuomotor Structure. In D. A Allport, ed. Language Perception and Production: Relationships Between Listening, Speaking, Reading and Writing. London: Academic Press, 1987.

Taub, Sarah F. (2001). Language From the Body: Iconicity and Metaphor in American Sign Language. Cambridge: Cambridge University Press.

Tervoort, Bernard.T. (1961). Esoteric Symbolism in the Communication Behavior of Young Deaf Children. American Annals of the Deaf, 106: 436-80.

Tordorov, Tzvetan (1982). Theories of the Symbol. Trans. Catherine Proter. Ithaca, New York: Cornell University Press.

Tylor, Edward B. (1865). Researches Into the Early History of Mankind and the Development of Civilization. London: John Murray.

Ultan, Russel (1978). Size-sound symbolism. In Jospeh H. Greenberg, ed. Universals of Human Language, Volume 2: Phonology. Stanford, CA: Stanford University Press.

van Ginneken, Jacques (1939). La Reconstruction Typologiques Des Langues Archaiques De L'humanité. Amsterdam.

Vermeerbergen, Myriam, Lorraine Leeson, and Onno Crasborn, eds. (2007). Simultaneity in Signed Languages: Form and Function. Amsterdam: John Benjamins.

Vico, Giambattista (1999 [1744]). New Science: Principles of the New Science Concerning the Common Nature of Nations. Third Edition, thoroughly corrected, revised and expanded by the author. Translated by David Marsh, with an Introduction by Anthony Grafton. Harmondsworth: Penguin Books.

Voeltz, F. K. Erhard and Christs Killian-Hatz, eds. (2001). Ideophones. Amsterdam: John Benjamins

Wallace, Alfred Russel. (1895). The Expressiveness of Speech Or Mouth-Gesture as a Factor in the Origin of Language. Fortnightly Review, 64: 528-543.

Waugh, Linda R. (2000). Against Arbitrariness: Imitation and Motivation Revived. In Patrizia Violi, ed. Phonosymbolism and Poetic Language. Turnhout, Belgium: Brepols, 25-56.

Werner, Heinz, and Bernard Kaplan (1963). Symbol Formation: An Organismic-Developmental Approach to Language and the Expression of Thought. New York: John Wiley and Sons.

Wescott, Roger W. (1971) Linguistic iconism. Language, 47: 416-428. 
Wilcox, Sherman E. (2004). Hands and Bodies, Minds and Souls: What Can Signed Languages Tell Us About the Origin of Signs? In Morana Alac, and Patrizia Violi, eds. In the Beginning: Origins of Semiosis. Semiotic and Cognitive Studies. Turnhout, Belgium: Brepols, 137-67.

Wilkins, David P. (2003). Why Pointing With the Index Finger is Not a Universal (in Cultural and Semiotic Terms). In Sotaro Kita, ed. Pointing: Where Language, Culture and Cognition Meet. Mahwah, New Jersey: Lawrence Erlbaum, 171-215.

Woll, Bencie.(2001). The Sign That Dares Not Speak Its Name: Echo Phonology in British Sign Language (BSL). In Penny Boyes-Braem, and R. Sutton-Spence, eds. The Hands Are the Head of the Mouth: The Mouth as Articulator in Sign Languages. Hamburg: SignumVerlag, 87-98. 